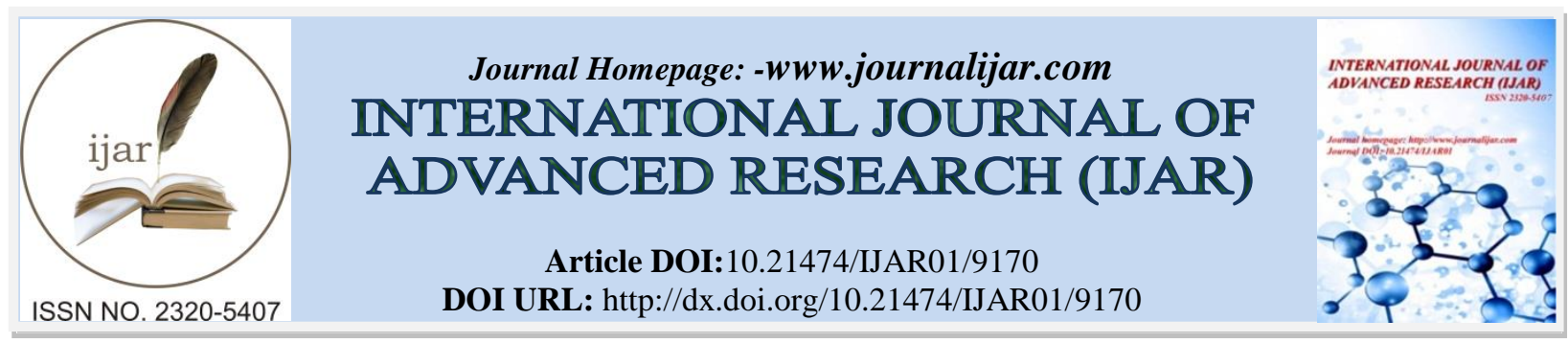

RESEARCH ARTICLE

\title{
PROBE OF PLASMA ASTROPHYSICS IN TRIPLE STAR SYSTEM
}

\author{
Ayan Kumar Makar \\ Centre of Plasma Physics - Institute of Plasma Research, Assam -782402, India
}

\section{Manuscript Info}

\section{Manuscript History}

Received: 24 March 2019

Final Accepted: 26 April 2019

Published: May 2019

Key words:-

Intraclustermedium,supernova, KELT-A, B and C, Cold fronts.
Abstract

When we consider universe, the most objects which are bound by gravity is the galaxy clusters. Intracluster medium forms the major component of space plasma. Most of the astronomical objects are build with matters which are ionised with high degree density of charges. These properties generates collective presence which are responsible for motion of charges in correspondence with varying electric and magnetic fields. In universe, most of the materials are in plasma state, mainly due to the constant and intense heating or collision which results in tear away of electrons. Astrophysical plasma generally incorporates the collection of starts which includes sun, gas between the stars radio galaxies and so on. To make it more precise, supernova remains to be an elegant example for astrophysical plasma. Nuclear physics remains to be the major source to understand these phenomenon in deep for carrying over with the research. Cold fronts in plasma galaxy clusters is an major research area which are mainly focussing on the front surface magnetic fields.

Similar to galaxy clusters, recent research trend is focussing on triple star system. Triple star systems basically offers better opportunity for researchers as it is evolving as an interesting research topic. This new research basically deals with informations related to gas giants, how mananging orbits regarding the closeness.KELT-A, B and C forms this three star system. The alignment and orbiting of these star determines the intensity between the orbits. The more distant the orbits is, the more will be the intensity. Considering these areas of research we focus on the triple star system in relation to plasma astrophysics.

Copy Right, IJAR, 2019,. All rights reserved.

\section{Introduction:-}

There have been growing interest for past few decades to explore the gravitationally bounded structure . These structures consisted of several objects held together by the force of gravity. For instance the super clusters of galaxies are the largest coherent structures in the present universe which accounts for the total masses of $\sim 10^{13}-$ $10^{15} \mathrm{M}_{\odot}$, just about $\geq 3-5 \%$ present in the proximate constellation .Some of the noteworthy parts of this constellation are the "intracluster medium" (ICM), a rarefied, highly ionized, metal rich, superheated plasma, which 
exists between galaxies in the cluster environment. Although the density of the cluster environment is extremely low , it accounts approximately for most of the baryonic materials in the galaxy clusters. Another significant element in the galaxy clusters is the dark matter, it is estimated that the majority of the matter is estimated to be composed of the dark matter which accounts for the $80 \%$ of the total mass, and about a quarter of its total energy density, the exact idea of which is at present obscure, however, is likely involved pitifully interacting non-baryonic particles, possibly being composed of some as-yet undiscovered subatomic particles. Intracluster medium(ICM) provides an unique opportunity to explore the space plasma which was established based on available hard X-ray data , temperature profiles of the galaxy clusters, and analyzing the chemical properties of ICM.

It was realized that ICM is enriched with heavy elements such as $\mathrm{O}, \mathrm{Ar}, \mathrm{Ca}, \mathrm{Mg}, \mathrm{Si}, \mathrm{Fe}$ and $\mathrm{Ni}$ at generally solar to $33 \%$ solar based bounties and with fully ionized helium and hydrogen plasma with densities between $10^{-4} \mathrm{~cm}^{-3}$ on the surface to a few $10^{-2} \mathrm{~cm}^{-3}$ in its core . Furthermore, intra-cluster medium is the tenuous X-ray emitting gas which emits radiation at $\mathrm{X}$ - ray wavelength by the process known as bremsstrahlung $($ brems $=$ brake, strahlung = radiation ) i.e. brake - radiation due to deceleration of charge particles, typically electrons, which has X-ray luminosities of $10^{36}$ to $10^{38} \mathrm{w}$ ( roughly billions to trillions times compared to the sun ). Aside from the most splendid outflow lines in the centers of bunches, the gas is optically slim to such X-beams. The noticeable distortion in the cosmic microwave background(CMB) spectrum due to inverse Compton scattering of thermal electrons in the ICM is known as SunyaevZeldovich impact (Sunyaev and Zeldovich 1972). Perceptions of radio sources in bunches furthermore, Faraday pivot estimations uncover that the ICM is pitifully charged (Carilli and Taylor 2002; Feretti et al. 2012), with attractive field qualities of $B \geq 0.1 \neq 10 \mu \mathrm{G}$, relating to an attractive weight which is about a couple of percents.The dispatch of the Chandra X-beam Observatory in 1999 gave an opportunity to peep into the material properties of the ICM, because of its $\geq 0.5$-arcsecond spatial goals. Be that as it may, notwithstanding for profound perceptions of the diffuse ICM emanation, practically the goals is diminished to a couple of arcseconds because of the requirement for adequate photon measurements. In any case, for close-by groups at separations of 16 to $300 \mathrm{Mpc}$ from us, rakish goals of 3 arcsec still relate to anticipated spatial goals on the sky of about $0.25 \mathrm{kpc}$ to $4 \mathrm{pcs}$, a scale tantamount to the molecule mean freeway. Among the primary bunch revelations by Chandra were sharp edges in surface splendor, first found in the groups A2142 (Markevitchet al.2000) and A3667 (Vikhlinin et al. 2001a). At furthest back, the edge had been already seen by ROSAT, however not with a similar detail (Markevitch et al. 1999). Such edges might be normal in impacting bunches, which should drive stuns into the ICM. Be that as it may, spectroscopic temperature estimations uncovered these edges were not stunning since the denser side of the irregularity is the cooler one. Due to this distinction, these edges were named "cold fronts". An all-encompassing audit of the properties and hypothesis of virus fronts in bunches was first given by Markevitch and Vikhlinin (2007, in the future MV07). Since that audit, a lot progressively cold fronts have been found in cosmic system bunches and comparative articles, e.g., universe gatherings and curved worlds. These frameworks are comparative as in with regards to this survey every one of them can be thought of as DM possibilities (of masses $\geq 10^{13} \neq 10^{15} \mathrm{M}_{\odot}$ ) loaded up with a hot plasma environment (of temperatures $\mathrm{T} \geq 10^{6} \neq 10^{8} \mathrm{~K}$ ).

\section{Literature Review}

Most of the astronomical objects are build with matters which are ionized with high degree density of charges. These properties generate collective presence which is responsible for the motion of charges in correspondence with varying electric and magnetic fields. In-universe, most of the materials are in a plasma state, mainly due to the constant and intense heating or collision which result in tear away of electrons. Astrophysical plasma generally incorporates the collection of stars which includes the sun, gas between the stars radio galaxies and so on. To make it more precise, supernova remains to be an elegant example for astrophysical plasma. Nuclear physics remains to be the major source to understand this phenomenon in deep for carrying over with the research. Cold fronts in plasma galaxy clusters is a major research area which is mainly focussing on the front surface magnetic fields.. Many of the most commonplace galactic articles comprise of issue that is ionized and has a sufficiently expansive thickness of charges to create aggregate conduct, or movement of numerous charges because of electric and attractive fields. Accordingly, these items are made out of the issue in a plasma state. Among a lot of instances of astrophysical plasmas are the environments of stars, including the Sun, the interstellar medium, or gas between the stars, gradual addition of plates around dark gaps, radio systems, and quasars. Immense measures of energy are discharged in numerous cosmic media, for example, supernova remainders. This energy discharge should drive irregular, fierce movements in the gas, similarly as we see on a progressively unobtrusive scale on Earth in tropical storms and tornadoes. The interstellar medium is the space between the stars. It shapes the air of the Milky Way Galaxy. The interstellar medium is loaded up with a gas having an enormous scope of densities and temperatures. Similar to galaxy clusters, the recent research trend is focussing on the triple star system. Triple star systems basically offer a 
better opportunity for researchers as it is evolving as an interesting research topic. This new research basically deals with the information related to gas giants, how managing orbits regarding the closeness.KELT-A, B, and C form this three-star system. The alignment and orbiting of these stars determine the intensity between the orbits.

\section{Problem statement}

In-universe, the vast majority of the materials are in the plasma state, chiefly because of the consistent and extraordinary warming or impact which result in tear away of electrons. Astrophysical plasma, for the most part, fuses the gathering of begins which incorporates sun, gas between the star's, radio cosmic systems and so on. To make it progressively exact, supernova stays to be an exquisite case for astrophysical plasma. Atomic material science stays to be the real source to comprehend these marvel in profound for continuing with the examination. Cold text styles in plasma cosmic system groups is a noteworthy research zone which is, for the most part, focussing on the textual style surface attractive fields. Similar to universal bunches, late research pattern is focussing on triple star framework. Triple star frameworks fundamentally offer a better open door for analysts as it is developing as a fascinating examination subject. This new research essentially manages information identified with gas mammoths, how managing orbits in regards to the closeness.KELT-A, B, and C frame this three-star framework. The arrangement and rotation of these stars determine the force between them. The more separate the contours are, the more will be the force. Considering these zones of research we center around the triple star framework in connection to plasma astronomy .

\section{Aim and objectives \\ 3.1 Aim}

To understand the features of probe of plasma dynamics in triple star system.

\subsection{Objectives}

- To discuss the recent simulations of cold fronts in galaxy clusters, focusing on those which have attempted to use these features to constrain ICM physics.

- To discuss the properties of probe of plasma astrophysics in triple star system

- To examine the effects of magnetic fields, viscosity, and thermal conductivity on the stability properties and long-term evolution of cold fronts.

\section{Proposed methodology}

Since the time when cold fronts were discovered a huge percentage of simulations have been carried out to model their formation and evolution in galaxy clusters. Here we are focused to discuss the limitations related to underlying plasma in galaxy clusters and how the plasma acts differently in the triple star system. A table showing the major simulations of cold fronts is discussed as follows.

\section{Table .1 - Major Simulations Of Cold Fronts}

\begin{tabular}{|c|c|c|c|c|c|c|c|}
\hline Reference & & $\begin{array}{l}\text { CF } \\
\text { Typ } \\
\text { e }\end{array}$ & Method & $\begin{array}{l}\text { Viscosit } \\
\mathbf{y}\end{array}$ & $\begin{array}{l}\text { Conductivit } \\
\mathbf{y}\end{array}$ & $\begin{array}{l}\text { Dimensionalit } \\
\mathbf{y}\end{array}$ & $\begin{array}{l}\Delta \text { minimu } \\
\mathbf{m ~ x}\end{array}$ \\
\hline Asai et al.(2004) & $\begin{array}{l}\text { (Asai- } \\
\text { MHD) }\end{array}$ & $\mathrm{R}$ & $\begin{array}{l}\mathrm{HD} / \mathrm{MH} \\
\mathrm{D}\end{array}$ & $\mathrm{N}$ & $\mathrm{I} / \mathrm{A}$ & 2D & $8.3 \mathrm{kpc}$ \\
\hline Asai et al.(2005) & $\begin{array}{l}\text { (Asai- } \\
\text { MHD) }\end{array}$ & $\mathrm{R}$ & $\begin{array}{l}\mathrm{HD} / \mathrm{MH} \\
\mathrm{D}\end{array}$ & $\mathrm{N}$ & $\mathrm{I} / \mathrm{A}$ & $3 \mathrm{D}$ & $8.9 \mathrm{kpc}$ \\
\hline Asai et al.(2007) & $\begin{array}{l}\text { (Asai- } \\
\text { MHD) }\end{array}$ & $\mathrm{R}$ & $\begin{array}{l}\mathrm{HD} / \mathrm{MH} \\
\mathrm{D}\end{array}$ & $\mathrm{N}$ & $\mathrm{I} / \mathrm{A}$ & $3 \mathrm{D}$ & $9.8 \mathrm{kpc}$ \\
\hline $\begin{array}{l}\text { Ascasibar\&Markevitch(200 } \\
6)\end{array}$ & (AM06 & $\mathrm{S}$ & $\begin{array}{l}\mathrm{HD} / \mathrm{MH} \\
\mathrm{D}\end{array}$ & $\mathrm{N}$ & $\mathrm{N}$ & $3 \mathrm{D}$ & $5 \mathrm{kpc}$ \\
\hline Dursi\&Pfrommer(2008) & & $\mathrm{R}$ & MHD & $\mathrm{N}$ & $\mathrm{N}$ & $3 \mathrm{D}$ & $0.016 \mathrm{R}$ \\
\hline Heinz et al. (2003) & & $\mathrm{R}$ & $\begin{array}{l}\mathrm{HD} / \mathrm{MH} \\
\mathrm{D}\end{array}$ & $\mathrm{N}$ & $\mathrm{N}$ & 2D cyl & $2 \mathrm{kpc}$ \\
\hline Roediger et al.(2011) & & $\mathrm{S}$ & HD & $\mathrm{N}$ & $\mathrm{N}$ & $3 \mathrm{D}$ & $0.5 \mathrm{kpc}$ \\
\hline Roediger et al.(2012 b) & R12 & $\mathrm{S}$ & HD & $\mathrm{N}$ & $\mathrm{N}$ & $3 \mathrm{D}$ & $1 \mathrm{kpc}$ \\
\hline Roediger et al.(2013 a) & R13 & $\mathrm{S}$ & HD & $\mathrm{I}$ & $\mathrm{N}$ & $3 \mathrm{D}$ & $0.5 \mathrm{kpc}$ \\
\hline
\end{tabular}




\begin{tabular}{|l|l|l|l|l|l|l|l|}
\hline Roediger et al.(2015 a ) & R15A & R & HD & N & N & 3D & $0.1 \mathrm{kpc}$ \\
\hline Roediger et al.(2015 b) & R15B & R & HD & I & N & 3D & $0.2 \mathrm{kpc}$ \\
\hline Suzuki et al.(2013) & S13 & R & $\begin{array}{l}\text { HD/MH } \\
\text { D }\end{array}$ & I/A & A & 3D & $9.1 \mathrm{kpc}$ \\
\hline ZuHone et al.(2010) & & $\mathrm{S}$ & HD & I & N & 3D & $5 \mathrm{kpc}$ \\
\hline ZuHone et al.(2011) & $\begin{array}{l}\text { ZML1 } \\
1\end{array}$ & $\mathrm{~S}$ & $\begin{array}{l}\text { HD/MH } \\
\text { D }\end{array}$ & N & N & 3D & $2 \mathrm{kpc}$ \\
\hline ZuHone et al.(2013) & Z13 & $\mathrm{S}$ & MHD & N & A & 3D & $2 \mathrm{kpc}$ \\
\hline ZuHone et al.(2015) & Z15 & $\mathrm{S}$ & $\begin{array}{l}\text { HD/MH } \\
\text { D }\end{array}$ & I/A & A & 3D & $1 \mathrm{kpc}$ \\
\hline
\end{tabular}

Here : $\mathrm{R}=$ Remnant-core, $\mathrm{S}=$ Sloshing

$\mathrm{N}=$ None , I = Isotropic, A = Anisotropic

Minimum resolution of simulation, for the "fiducial" simulation in the study.

Simulation performed in arbitrary units, $\mathrm{R}$ is the radius of the core.

At first, the fundamental material science has hidden these distraction. As said earlier, due to the way that the electron and particle mean collisions are smaller than the length scales under thought, the ICM is normally displayed as a warm, charged plasma in the liquid estimate. Nonetheless, as opposed to the standard MHD requesting of scales, the Larmor radii of the particles are smaller than their mean collisions, where the Larmor radii are on the request of NPC contrasted with the kpc size of the mean collisions. In this way, a given molecule revolves commonly around the neighborhood attractive field contour before experiencing another molecule. Because of the little Larmor radii and the relative shortcoming of crashes, we may find that the opposite and parallel weights of the particles and the vehicle coefficients of diffusive procedures are anisotropic with regard to the nearby course of the attractive field line. In this circumstance, the anisotropy of the opposite and parallel weights is created by the preservation of the first furthermore, second adiabatic invariants of the particles. Under these suspicions, the liquid portrayal of the ICM can be recorded in terms of the Braginskii-MHD conditions (Braginskii 1965, displayed here in traditionalist structure, and in Gaussian units). The equations for conservation of mass, momentum, energy, and magnetic field are:

$$
\begin{aligned}
& \frac{\partial \rho}{\partial t}+\nabla \cdot(\rho v)=0 \\
& \frac{\partial(\rho v)}{\partial t}+\nabla \cdot(\rho v v+P)=\rho g \\
& \frac{\partial(\rho e)}{\partial t}+\nabla \cdot[(\rho e l+P) \cdot v+Q]=\rho g \cdot v \\
& \frac{\partial B}{\partial t}+\nabla \cdot(v B-B v)=0
\end{aligned}
$$

Where the total(thermal and magnetic) pressure tensor is represented as

$P=p \perp I-(p \perp-p \|) \widehat{b} \hat{b}+\frac{B^{2}}{8 \pi} I-\frac{B B}{4 \pi}$

Here $\widehat{b}=B / B$ represents the unit vector in the direction of the local magnetic field and the total thermal pressure that satisfies 
$p e=\frac{\rho v^{2}}{2}+\rho \in+\frac{B^{2}}{8 \pi}$

In the above mentioned equations $\boldsymbol{\rho}$ represents the gas density, $\boldsymbol{v}$ represents the fluid velocity, $\in$ represents the gas thermal energy per unit volume, $\mathbf{B}$ is the magnetic field vector, $\mathbf{g}$ is the gravitational acceleration, $\mathbf{Q}$ is the heat flux vector and $\mathbf{I}$ represents the unit dyad.

An adiabatic equation of state of an Ideal gas $p=(\gamma-1) \rho \in$ where $\gamma=5 / 3$ is taken for assumption along with same amount of electron and ion temperatures and abundant amount of $\mathrm{H}$ and $\mathrm{He}$ with traces of metals for the average molecular weight of $\bar{A} \approx 0.6$.

The vast majority of astrophysical literature on galaxy clusters does not present the MHD conditions with a weight anisotropy, and rather depicts this impact regarding a gooey transition. This methodology presently demonstrates how these two portrayals are identical. Contrasts in the two segments of the warm weight emerge from the preservation of the first and second adiabatic invariants for every molecule on timescales and a lot more noteworthy than the converse of the ion gyrofrequency, $\Omega_{\mathrm{g}}{ }^{-1}$. Chew et al. (1956). At the point when the particle impact recurrence $v_{i i}$ is a lot bigger than the rates of progress everything being equal, a condition for the weight anisotropy can be acquired by adjusting its generation by adiabatic invariance with its unwinding through impacts (cf.Schekochihin et al. 2005):

$p_{\perp}-p_{\|}=0.960 \frac{p_{i}}{v_{i i}} \frac{\partial}{\partial t} \ln \left(\frac{B^{3}}{\rho^{2}}\right)$

$\mathbf{p}_{\mathbf{i}}$ represents the thermal pressure of the ions.

The dynamic viscosity coefficient for the ions, or the "Spitzer" viscosity, is given by (Spitzer 1962; Braginskii 1965; Sarazin 1988):

$\mu_{S_{p}}=0.960 \frac{p_{i}}{v_{i i}} \approx 2.2 \times 10^{-15} \frac{T^{5 / 2}}{\ln \left(\lambda_{i}\right)} \mathrm{gcm}^{-1} \mathrm{~s}^{-1}$

The temperature $\mathbf{T}$ is in Kelvin, $\boldsymbol{V}_{\mathbf{i i}}$ is the ion-ion collision frequency and $\ln \left(\boldsymbol{\lambda}_{\mathbf{i}}\right) \approx \mathbf{4 0}$.

$Q=-k_{S_{p}} \widehat{b} \widehat{b \cdot \nabla T}$.

$k_{S_{p}}=\frac{3.2 k_{B} p_{e}}{m_{e} v_{e e}} \approx 1.84 \times 10^{-5} \frac{T^{5 / 2}}{\ln \left(\lambda_{e}\right)} \operatorname{erg~cm}^{-1} S^{-1} K^{-1}$

$\mathbf{p}_{\mathbf{e}}$ represents the thermal pressure of the electrons, $\boldsymbol{V}_{\mathbf{e e}}$ is the electron-electron collision frequency, and $\ln \left(\lambda_{\mathrm{e}}\right) \approx 40$. The heat flux due to ion collisions is negligible because of the larger mass and hence it is neglected.

Up until now, most recreations of the ICM have not worked with the full arrangement of these conditions, yet rather in certain physically-roused cut-off points . Specifically, there have been two approximations of these conditions in basic use. In the point of confinement that the weight anisotropy is small, we recuperate standard MHD. In this survey, various works in this MHD limit are discussed, which accentuate the impact of the attractive field on virus fronts. Since the Prandtl number of the Spitzer depiction of the ICM is extremely small, the warm diffusivity overwhelms over the energy diffusivity:

$\operatorname{Pr}=\frac{v}{X}=0.5 \frac{\ln \lambda_{e}}{\ln \lambda_{i}}\left(\frac{2 m_{e}}{m_{i}}\right)^{1 / 2} \simeq 0.02$ 
Accordingly, another farthest point of these conditions considers MHD with warm conductivity is also included. We will likewise consider various recreations that have worked in this breaking point. The second utmost of these conditions that is regularly demonstrated is that in which the attractive field is extremely powerless or violent all through the volume of the group. For this situation, however, the impacts of magnetic pressure and tension are neglected, the anisotropy of the heat and momentum fluxes show itself as effective concealment of the transport coefficients when averaged over a small volume, expecting the magnetic field to be isotropically tangled.We may then re-compose Equations as following:

$$
\begin{aligned}
& \frac{\partial(\rho v)}{\partial t}+\nabla \cdot(\rho v v+p l+\pi)=\rho g \\
& \frac{\partial(\rho e)}{\partial t}+\nabla \cdot\{(\rho e+\rho) l+\pi\} . v+Q=\rho g \cdot v
\end{aligned}
$$

Where the conductive and viscous flux terms may be written as :

$$
\begin{aligned}
& \mathrm{Q}=-\mathrm{f}_{\mathrm{C}} \mathrm{K}_{\mathrm{SP}} \nabla \mathrm{T} \\
& \Pi=-\mathrm{f}_{\mathrm{V}} \mu_{S P} \nabla \mathrm{v}
\end{aligned}
$$

where the "suppression factors" $f_{C}$ and $f_{v}$ represent the diminished conductivity and thickness as a result of the averaging over the arbitrary heading of the attractive field, or other microscale forms that stifle the powerful conductivity as well as thickness beneath the Spitzer esteem. This HD farthest point of the conditions is the assumption that has been accepted in various recreation works, including some imperative ones considered in this survey. Concept of this breaking point is pertinent, since the limited Larmor sweep consequences for the vehicle coefficients may frequently just uncover themselves in a spatially arrived at the midpoint of the way because of projection impacts and the limited goals of $\mathrm{X}$-beam perceptions.

\section{Significance:-}

Till now a lot of research work has been carried out in the field of plasma for galaxy clusters. The recent research trend is focussing on the triple star system. Triple star systems basically offer a better opportunity for researchers as it is evolving as an interesting research topic. This new research basically deals with information related to gas giants, how managing orbits regarding the closeness.KELT-A, B, and $\mathrm{C}$ form this three-star system. The alignment and orbiting of these stars determine the intensity between the orbits. The more distant the orbits are, the more will be the intensity. Thus considering all the possibilities it offers an unique opportunity to explore the branch of plasma astrophysics with the help of triple stars systems.

\section{References:-}

1. Peynot, Thierry, et al. "Autonomous reconfiguration of a multi-modal mobile robot."Workshop on Automated Diagnosis, Repair and Re-Configuration of Robot Systems, IEEE International Conference on Robotics and Automation (ICRA). 2011.

2. Cherubini, Andrea, et al. "A multimode navigation system for an assistive robotics project."Autonomous Robots 25.4 (2008): 383-404.

3. Dongyue, Qu, Hu Yuanhang, and Zhang Yuting. "The investigation of the obstacle avoidance for mobile robot based on the multi sensor information fusion technology."Int. J. Mat. Mech. Manuf 1 (2013): 366-370.

4. Saldana, David, Renato Assunçao, and Mario FM Campos. "A distributed multi-robot approach for the detection and tracking of multiple dynamic anomalies."2015 IEEE International Conference on Robotics and Automation (ICRA). IEEE, 2015.

5. Mardani, Arman, and SaeedEbrahimi. "A Novel multimode mobile robot with adaptable wheel geometry for maneuverability improvement."International Journal of Robotics, Theory and Applications 4.4 (2016): 1-15.

6. Zhang, Jinzhu, Zhenlin Jin, and HaibingFeng. "Type synthesis of a 3-mixed-DOF protectable leg mechanism of 
a firefighting multi-legged robot based on GF set theory."Mechanism and Machine Theory 130 (2018): 567584.

7. Li, Yezhuo, Yan-an Yao, and Yanying He. "Design and analysis of a multi-mode mobile robot based on a parallel mechanism with branch variation."Mechanism and Machine Theory 130 (2018): 276-300.

8. Nadon, Félix, Angel Valencia, and Pierre Payeur. "Multi-Modal Sensing and Robotic Manipulation of NonRigid Objects: A Survey."Robotics 7.4 (2018): 74.

9. Zhang, ZiQiang, et al. "Mechanism design for locust-inspired robot with one-DOF leg based on jumping stability."Mechanism and Machine Theory 133 (2019): 584-605.

10. Michaud, François, and Maja J. Matarić. "Learning from history for behavior-based mobile robots in nonstationary conditions."Machine Learning 31.1-3 (1998): 141-167.

11. Pandilov, Zoran, and Vladimir Dukovski. "COMPARISON OF THE CHARACTERISTICS BETWEEN SERIAL AND PARALLEL ROBOTS."ActaTechnicaCorvininesis-Bulletin of Engineering 7.1 (2014).

12. Sarfraz, Hassan. Kinematics and Optimal Control of a Mobile Parallel Robot for Inspection of Pipe-like Environments. Diss. Universitéd'Ottawa/University of Ottawa, 2014.

13. Rasheed, Tahir, et al. "Kinematic modeling and twist feasibility of mobile cable-driven parallel robots."International Symposium on Advances in Robot Kinematics. Springer, Cham, 2018.

14. Yang, Hai, et al. "On the design of mobile parallel robots for large workspace applications."IDETC'11: International Design Engineering Technical Conferences Computers and Information in Engineering Conference. ASME, 2011.

15. Wang, Z-Y., X-L. Ding, and Alberto Rovetta. "Analysis of typical locomotion of a symmetric hexapod robot."Robotica28.6 (2010): 893-907.

16. Sproewitz, Alexander, et al. "Learning to move in modular robots using central pattern generators and online optimization."The International Journal of Robotics Research27.3-4 (2008): 423-443.

17. Cavaliere, A. G., Gursky, H. \& Tucker, W. H. 1971 Extragalactic X-ray Sources andAssociations of Galaxies. Nature 231, 437-438.

18. Carilli, C. L. \& Taylor, G. B. 2002 Cluster Magnetic Fields. Annu. Rev. Astron. Astrophys.40, 319-348 . 